\title{
AVERAGES OF CONTINUOUS FUNCTIONS ON COUNTABLE SPACES ${ }^{1}$
}

\author{
BY MELVIN HENRIKSEN AND J. R. ISBELL
}

Communicated by Walter Rudin, November 18, 1963

Introduction. Let $X=\left\{x_{1}, x_{2}, \cdots\right\}$ be a countably infinite topological space; then the space $C^{*}(X)$ of all bounded real-valued continuous functions $f$ may be regarded as a space of sequences $\left(f\left(x_{1}\right), f\left(x_{2}\right), \cdots\right)$. It is well known [7, p. 54] that no regular (Toeplitz) matrix can sum all bounded sequences. On the other hand, if $\left(x_{1}, x_{2}, \cdots\right)$ converges in $X$ (to $x_{m}$ ), then every regular matrix sums all $f$ in $C^{*}(X)$ (to $f\left(x_{m}\right)$ ).

The main result of this paper is that if a regular matrix sums all $f$ in $C^{*}(X)$ then it sums $f$ to $\sum \alpha_{n} f\left(x_{n}\right)$, for some absolutely convergent series $\sum \alpha_{n}$. We use this to show that no regular matrix can sum all of $C^{*}(X)$ if $X$ is extremally disconnected (the closure of every open set is open). This extends a theorem of W. Rudin [6], which has an equivalent hypothesis ( $X$ is embeddable in the Stone-Cech compactification $\beta N$ of a discrete space) and concludes that not all $f$ in $C^{*}(X)$ are Cesàro summable.

For any continuous linear functional $\phi$ on $C^{*}(X)$ one has a ("Riesz") representation $\phi(f)=\int f d \mu$, where $\mu$ is a Radon measure on $\beta X$. Our main result is just that $X$ supports $\mu ; \mu$ is forced to be atomic since $X$ is countable. We show further that $X$ has a subset $T$, the set of heavy points, such that the functionals we are concerned with correspond exactly to measures $\mu$ supported by $T$ with $\mu(T)=1$. Our knowledge of $T$ is limited; it will be summarized elsewhere.

1. Representation. It is well known [7, p. 57] that a matrix $A=\left(a_{i j}\right)$ defines a regular summability method if and only if it satisfies the conditions (1) $\sum_{j} a_{i j}=1+o(1)$, (2) $\sum_{j}\left|a_{i j}\right|$ is uniformly bounded, and (3) for each $j, a_{i j} \rightarrow 0$.

For all the present results on real-valued functions, we may assume without loss of generality that our topological spaces are completely regular. Then each countable space $X$ has a base of open-and-closed sets, and each $f \in C^{*}(X)$ is a uniform limit of linear combinations of characteristic functions of these basic sets.

Suppose that $A$ is a regular matrix such that $\phi_{A}(f)$ $=\lim _{i \rightarrow \infty} \sum_{j} a_{i j} f\left(x_{j}\right)$ exists for each $f \in C^{*}(X)$. For each open-andclosed subset $U$ of $X$, let $c_{U}$ denote its characteristic function, and let

1 This research was supported by the National Science Foundation. 
$a(U)=\phi_{A}\left(c_{U}\right)$. Also, let $b(U)=\sup \sum\left|a\left(V_{i}\right)\right|$, where $\left\{V_{i}\right\}$ ranges over all finite disjoint families of open-and-closed subsets of $U$. A bound for $\sum_{j}\left|a_{i j}\right|$ is a bound for $b(U)$; also $a$ and $b$ are finitely additive.

For each point $x \in X$, let $\alpha(x)$ be the limit of $a(U)$ over the filter base $\mathfrak{u}_{x}$ of open-and-closed neighborhoods of $x$. It exists since the monotone function $b(U)$ converges, which implies that

$$
\begin{aligned}
& \lim \left\{b\left(U \sim U^{\prime}\right): U, U^{\prime} \in \mathcal{U}_{x}, U^{\prime} \subset U\right\}=0, \\
& \text { so }\left|a(U)-a\left(U^{\prime}\right)\right|=\left|a\left(U \sim U^{\prime}\right)\right| \leqq b\left(U \sim U^{\prime}\right) .
\end{aligned}
$$

Lemma. The series $\sum \alpha\left(x_{n}\right)$ is absolutely convergent with sum 1.

Proof. For any $\epsilon>0$, there exist $U_{n} \in \mathfrak{u}_{x_{n}}$, for $n=1,2, \cdots$, such that for any $V_{n} \in \mathfrak{u}_{x_{n}}$ satisfying $V_{n} \subset U_{n}, \sum b\left(U_{n} \sim V_{n}\right)<\epsilon$. Thus $\left|\sum a\left(U_{n}\right)-\sum \alpha\left(x_{n}\right)\right| \leqq \epsilon$, and with a further error of $\epsilon$, we can replace the sets $U_{n}$ by a disjoint family $\left\{W_{n}\right\}$ covering $X$. Then absolute convergence is evident; and if $\sum \alpha\left(x_{n}\right) \neq 1$, we may choose $\epsilon>0$ so small that $\sum a\left(W_{n}\right)=1-d$ with $d \neq 0$.

Let $a_{i j}^{*}=\sum\left[a_{i k}: x_{k} \in W_{j}\right]$; note that $\lim _{i} a_{i j}^{*}=a\left(W_{j}\right)$. Let

$$
c_{i j}=\frac{a_{i j}^{*}-a\left(W_{j}\right)}{d} \text {. }
$$

Then $\left(c_{i j}\right)$ is regular since (1) and (3) hold, and $\sum_{j}\left|c_{i j}\right|$ is bounded by $2 /|d|$ times the bound for $\sum_{j}\left|a_{i j}\right|$. Since no regular matrix can sum all sequences of zeros and ones $[7$, p. 54], there is a subset $Z$ of $N$ such that $\sum_{j \in Z} c_{i j}$ does not converge, so $W=\bigcup\left\{W_{n}: n \in Z\right\}$ is an open-and-closed set for which $a(W)$ does not exist. This contradiction establishes the lemma.

Corollary. For any open-and-closed set $U, \sum[\alpha(x): x \in U]=a(U)$.

Proof. Passing to $(X \sim U)$ if necessary, we may assume that $a(U) \neq 0$. The matrix $\left(b_{i j}\right)$ obtained by letting $b_{i j}=a_{i j} / a(U)$ if $x_{j} \in U$, and by letting $b_{i j}=0$ otherwise, is a regular matrix that sums each element of $C^{*}(U)$, so the lemma applies.

THEOREM 1. If a regular matrix summability method $\phi$ sums all bounded continuous functions on a countably infinite topological space $X=\left\{x_{1}, x_{2}, \cdots\right\}$, then there is an absolutely convergent series $\sum \alpha_{n}$ with sum 1 such that for each $f \in C^{*}(X), \phi(f)=\sum \alpha_{n} f\left(x_{n}\right)$.

Proof. The corollary shows this for characteristic functions and the rest follows from linearity and continuity. 
2. Reduction to points. As indicated in the introduction, we can reduce the problem of which functionals $f \rightarrow \sum \alpha_{n} f\left(x_{n}\right)$ are given by regular matrices to the problem for single points, $f \rightarrow f(x)$. There is a further reduction to the case that $x$ is the only nonisolated point. (Obviously $x$ must be nonisolated.) We define a heavy point $x$ of a countable space $\left\{x_{1}, x_{2}, \cdots\right\}$ as one such that there exists a regular matrix $A$ such that for every bounded function $f$ continuous at $x$, $\phi_{A}(f)=f(x)$.

Theorem 2. A functional $\phi(f)=\sum \alpha_{n} f\left(x_{n}\right)$ on $C^{*}(X)$ is representable as $\phi_{A}$ for some regular matrix $A$ if and only if $\sum \alpha_{n}=1$ and $\alpha_{n}=0$ whenever $x_{n}$ is not a heavy point.

The proof will be published elsewhere, together with the results abstracted in [4], which tell a little about heavy points. It is easy to see that the limit of a convergent (nonconstant) sequence is a heavy point; another heavy point that is not the limit of a sequence is exhibited, essentially, in [3, Example 3.3].

3. Removable points. A point $x$ for which every function $f \in C^{*}(X \sim\{x\})$ has an extension in $C^{*}(X)$ cannot be a heavy point; for the matrix $A$ summing $C^{*}(X)\left(\phi_{A}(f)=f(x)\right)$ would, with one column deleted, sum all of $C^{*}(X \sim\{x\})$ ( $\phi_{A}$ violating Theorem 1). As the omitted proof of Theorem 2 is long, we note that this argument works as well with $\phi_{A}(f)=\sum \alpha_{n} f\left(x_{n}\right)$, if $x=x_{r}$ has a nonzero coefficient $\alpha_{r}$; that is, Theorem 1 suffices. Moreover, there is a trifle of extra information; if $A$ sums every $f$ in $C^{*}(X)$ to $\sum \alpha_{n} f\left(x_{n}\right)$, and $\alpha_{m} \neq 0$, then there is a bounded function discontinuous only at $x_{m}$ that $A$ fails to sum.

A subspace $Y$ of a completely regular space $X$ is said to be $C^{*}$ embedded if every $f \in C^{*}(Y)$ has an extension in $C^{*}(X)$. It is well known [2, p. 23] that a space $X$ is extremally disconnected if and only if each of its dense subspaces is $C^{*}$-embedded. Thus, from Theorem 1 and the above, we have

THeOREM 3. If the complement of each point of a countably infinite space $X$ is $C^{*}$-embedded, in particular, if $X$ is extremally disconnected, then no regular matrix can sum every element of $C^{*}(X)$.

The complement of every point of $X$ may be $C^{*}$-embedded without $X$ being extremally disconnected. For example, identify two copies of a countable extremally disconnected space along a closed dense in itself subspace.

In [6], W. Rudin proved that if $X$ is a countable subspace of $\beta N$, 
there is an $f \in C^{*}(X)$ that is not $C_{1}$-summable. Any such $X$ is extremally disconnected; indeed every subspace of a countable subspace of $\beta N$ is $C^{*}$-embedded [2, p. 97]. Every countable extremally disconnected space takes this form; in fact

Every extremally disconnected space $X$ having a dense subspace of power $\mathfrak{m}$ can be embedded in $\beta D$, where $D$ is a discrete space of power $\mathfrak{m}$.

PRoof. There is a mapping $\tau$ of $D$ onto a dense subspace $Y$ of $X$ which has a continuous extension over $\beta D$ onto $\beta X[2, \mathrm{p}$. 86]. Let $E$ be a closed subspace of $\beta D$ minimal with respect to the property of being mapped onto $\beta X$ by $\tau$. Gleason shows in [1] that the restriction of $\tau$ to $E$ is a homeomorphism since $\beta X$ is extremally disconnected [2, p. 96].

This easy application of Gleason's theorem answers a question of Katětov, who asked if every extremally disconnected space, every subspace of which is normal, can be embedded in $\beta D$ for some discrete space $D[5]$.

\section{REFERENCES}

1. A. Gleason, Projective topological spaces, Illinois J. Math. 2 (1958), 482-489.

2. L. Gillman and M. Jerison, Rings of continuous functions, Van Nostrand, New York, 1960.

3. M. Henriksen, Multiplicative summability methods and the Stone-Cech compactification, Math. Z. 71 (1959), 427-435.

4. M. Henriksen and J. R. Isbell, Multiplicative summability methods and the Stone-Čech compactification. II, Notices Amer. Math. Soc. 11 (1964), 90.

5. M. Katětov, On nearly discrete spaces, Časopis Pěst. Mat. Fys. 75 (1950), 69-78.

6. W. Rudin, Averages of continuous functions, Duke Math. J. 25 (1958), 197-204.

7. K. Zeller, Theorie der Limitierungsverfahren, Ergebnisse der Mathematik und ihrer Grenzgebeite, Springer, Berlin, 1958.

The Institute for Advanced Study, Purdue University and

The University of Washington 